\section{A role for a novel 'trans-pseudoknot' RNA-RNA interaction in the functional dimerization of human telomerase}

\author{
Hinh Ly, ${ }^{1,3}$ Lifeng $\mathrm{Xu}^{2,3}$ Melissa A. Rivera, ${ }^{2}$ \\ Tristram G. Parslow, ${ }^{1}$ and \\ Elizabeth H. Blackburn ${ }^{2,4}$ \\ ${ }^{1}$ Departments of Pathology, Microbiology, and Immunology, \\ and ${ }^{2}$ Department of Biochemistry and Biophysics, University \\ of California, San Francisco, California 94143, USA
}

The integral RNA (hTER) of the human telomerase ribonucleoprotein has a conserved secondary structure that contains a potential pseudoknot. Here we examine the role of an intermolecular hTER-hTER interaction in the previously reported functional dimerization of telomerase. We provide evidence that the two conserved, complementary sequences of one stem of the hTER pseudoknot domain can pair intermolecularly in vitro, and that formation of this stem as part of a novel "transpseudoknot" is required for telomerase to be active in its dimeric form. Such RNA-RNA interaction mirrors a known property of retroviral reverse transcriptases, which use homodimeric viral genomic RNA substrates.

Supplemental material is available online at http://www. genesdev.org.

Received November 21, 2002; revised version accepted March 3, 2003.

A small portion of telomerase RNA is used as the template for synthesizing the short, tandemly repeating DNA sequences characteristic of telomeres (for reviews, see Nugent and Lundblad 1998; Blackburn 2000; Blasco 2002). The catalytic protein component of telomerase, called TERT, is structurally and evolutionarily related to other reverse transcriptases (Lingner et al. 1997; Nakamura and Cech 1998), but is distinct in that it associates stably with the telomerase RNA. The active telomerase complex has previously been reported to comprise at least two copies each of telomerase RNA and of TERT protein (Prescott and Blackburn 1997a,b; Beattie et al. 2000; Wenz et al. 2001), along with several accessory factors (for reviews, see Nugent and Lundblad 1998; Blackburn 2000; Blasco 2002). The molecular interactions required for the enzymatic function of this dimeric complex have not been fully determined.

Although telomerase RNAs differ widely in overall

\footnotetext{
${ }^{3}$ These authors contributed equally to this work. ${ }^{4}$ Corresponding author.

E-MAIL telomer@itsa.ucsf.edu; FAX (415) 514-2913.

Article and publication are at http://www.genesdev.org/cgi/doi/10.1101/ gad.1060803.

[Keywords: Human telomerase; telomerase RNA; retroviral genomic RNA; telomerase RNA pseudoknot; telomerase enzymatic activity]
}

length and sequence among species, phylogenetic comparisons suggest that those from protozoa and vertebrates each have a conserved core secondary structure that includes a pseudoknot (for review, see Blackburn 2000, Chen et al. 2002). The phylogenetic conservation of this pattern in close proximity to the template, and the strict conservation of $\mathrm{P} 3$ sequence among vertebrates, together strongly suggest that the pseudoknot region is biologically important, as does the finding that telomerase enzymatic function can be severely impaired by naturally occurring (Vulliamy et al. 2001; Comolli et al. 2002) or engineered (Gilley and Blackburn 1999. Bachand and Autexier 2001; Martin-Rivera and Blasco 2001) mutations that interfere with the potential for pseudoknot formation.

It was previously reported that individual copies of the 1300-base telomerase RNA molecule of the yeast Saccharomyces cerevisiae can interact functionally (Prescott and Blackburn 1997a,b). This was based on the finding that a specific mutant telomerase RNA carrying a three-base substitution in its template sequence lacked any biological activity when expressed alone but regained its templating function when coexpressed with the wild-type RNA (Prescott and Blackburn 1997a). Significantly, the resulting telomeric DNAs were made up of densely interspersed wild-type and mutant repeats, implying that telomerase may readily alternate between at least two RNA templates during synthesis of a single telomere (Prescott and Blackburn 1997b).

Those findings recalled the template-switching characteristic of retroviral RTs. Retroviruses normally package two identical copies of single-stranded genomic RNA into each virion, and their RTs alternate repeatedly between these two templates during synthesis of a DNA provirus (Coffin 1979; Hu and Temin 1990). Template switching is facilitated by the inherent ability of retroviral genomic RNAs to form parallel, noncovalent homodimers, with the most thermostable and conserved dimer contacts occurring in the $5^{\prime}$ leader region near the site where reverse transcription begins. In all retroviral genomes studied to date, these $5^{\prime}$ contacts are created by one or two short RNA stem-loops that initially hybridize to the opposite strand through sequences in their loops (Laughrea and Jette 1994; Paillart et al. 1996; Clever and Parslow 1997; Ly and Parslow 2002). Such dimer-initiating stem-loops are defined experimentally by their unique efficiency in promoting dimerization of highly structured, protein-free RNAs in vitro, and by this reaction's dependence on mild heating that enables the stem-loops to refold (Clever and Parslow 1997; Ly and Parslow 2002).

Like telomerase RNAs of many eukaryotes, hTER contains a predicted pseudoknot-a stem-loop whose loop can potentially base-pair with an adjacent region in the same RNA molecule to form a short helix called P3 (Fig. 1A; for review, see Blackburn 2000; Chen et al. 2000). Here we report that $\mathrm{P} 3$ base-pairing can also occur intermolecularly, leading to spontaneous assembly of hTER dimers in vitro, and that pairs of inactive hTER mutants with complementary P3 mutations can dimerize and regain biological activity when mixed. Our findings imply a novel quaternary structure for catalytically active hTER, and suggest that template RNA dimerization may be a general feature of reverse transcription. 


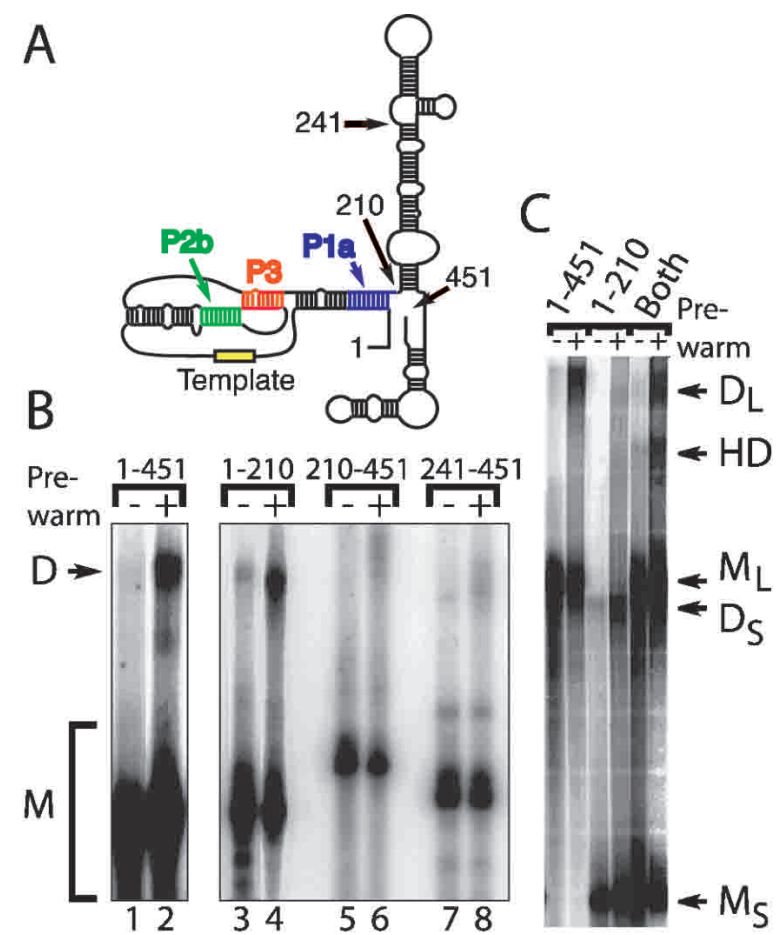

Figure 1. Sequences in the $5^{\prime}$ half of human telomerase RNA (hTER) mediate efficient RNA dimerization in vitro. (A) Proposed secondary structure (Chen et al. 2000) of the 451-base hTER. Specific residues are numbered. The templating sequence (yellow rectangle) and the putative paired regions $\mathrm{P} 1 \mathrm{a}, \mathrm{P} 2 \mathrm{~b}$, and $\mathrm{P} 3$ are shown. (B) Synthetic RNAs representing the indicated portions of hTER were denatured, snap-cooled, incubated on ice $(-)$ or at $37^{\circ} \mathrm{C}(+)$, and analyzed by nondenaturing gel electrophoresis. $(C)$ Full-length hTER and its $5^{\prime}$ half form a heterodimer when mixed. RNAs were analyzed, either individually or in equimolar mixture, as described for panel $B$. The heterodimer $(H D)$, and the monomers $\left(M_{S}\right.$ and $\left.M_{L}\right)$ and homodimers $\left(D_{S}\right.$ and $\left.D_{L}\right)$ of the short and long RNAs, respectively, are indicated.

\section{Results and Discussion}

We asked whether synthetic hTER forms dimers spontaneously under the conditions known to promote retroviral genomic dimerization. Radiolabeled full-length hTER (451 bases; Fig. 1A) was synthesized, briefly denatured at $95^{\circ} \mathrm{C}$, refolded on ice, and then examined by nondenaturing gel electrophoresis either with or without preheating to $37^{\circ} \mathrm{C}$ for $2 \mathrm{~h}$. As shown in Figure $1 \mathrm{~B}$, essentially all of the full-length RNA migrated as monomers $(\mathrm{M})$ when unheated (lane 1). Upon preheating to $37^{\circ} \mathrm{C}$ (Fig. 1B, lane 2), however, $20 \%-40 \%$ converted to a discrete, lower-mobility species suggestive of a dimer (D). A truncated RNA comprising only the 5' half of hTER likewise formed an apparent dimer (Fig. 1B, lanes 3,4 ), whereas two different 3 ' fragments of similar length and at similar concentrations did not (Fig. 1B, lanes 5-8). The dimeric nature of the complexes was confirmed by the finding that, when incubated together (Fig. 1C), the full-length and 5 '-half RNAs gave rise not only to their respective homodimers $\left(D_{L}\right.$ and $\left.D_{S}\right)$ but also to the predicted single, intermediate-sized heterodimer (HD). No bands suggesting higher-order oligomers were detected in this or any additional analyses (Supplementary Fig. 1). Collectively, these results indicated that hTER dimerizes efficiently at physiologic temperature in vitro, and that sequences in the $5^{\prime}$ half of hTER are necessary and sufficient for dimer formation.

The proposed secondary structure (Chen et al. 2000) of the $5^{\prime}$ half of hTER includes the single-stranded template sequence as well as three paired regions designated P1P3, with P2 and P3 forming the stem and loop helices of the pseudoknot, respectively (Fig. 1A). We introduced a series of clustered point mutations into each of these regions of hTER individually. As shown in Figure 2A, none of the tested mutations in the template sequence or in the distal parts of P1 or P2 (denoted P1a and P2b, respectively) had any appreciable effect on in vitro dimer yield. In contrast, dimerization was markedly reduced by three extensive P3 mutations. Two of these, designated P3-A-up and P3-A-dn, each fully substituted the upper and lower P3 strands, respectively, and were complementary to each other. When the P3-A-up and P3-A-dn

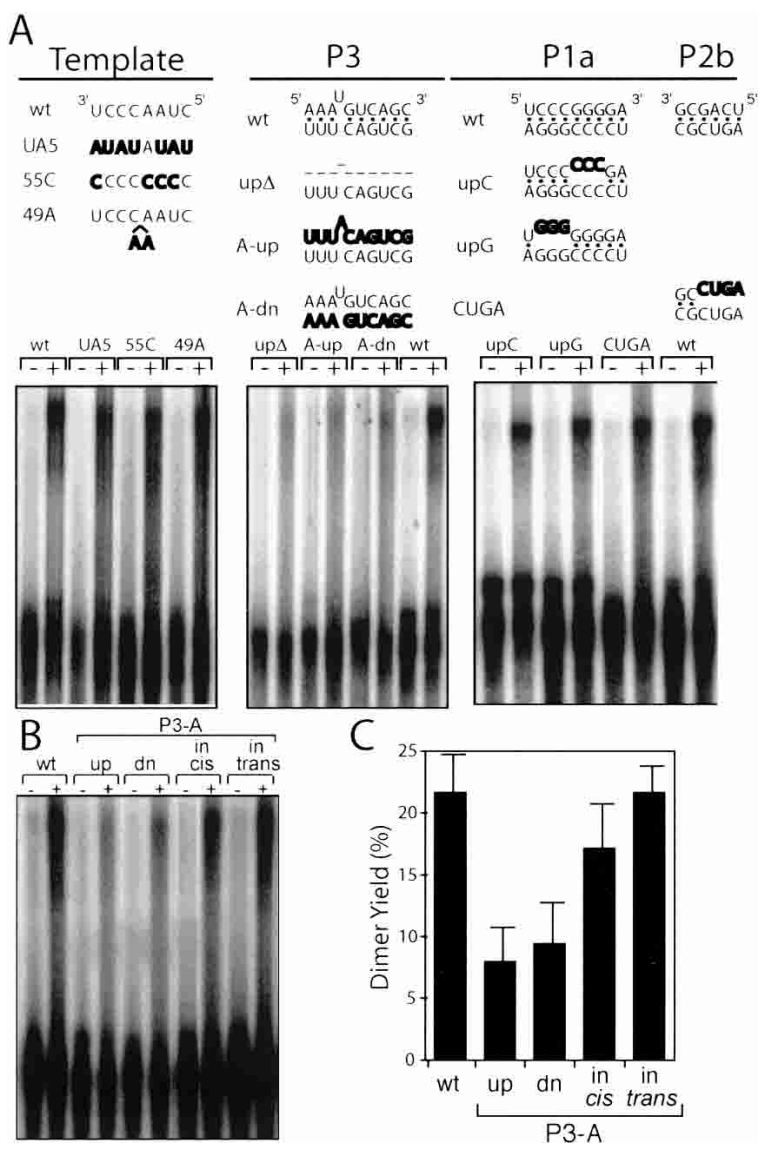

Figure 2. Sequence complementarity at P3 is required for hTER dimerization. (A) Mutations in P3 selectively abrogate dimer formation in vitro. Dimerization reactions were performed as described for Figure 1, using synthetic wild-type (wt) or mutant forms of hTER residues $1-451$. The template sequence and paired regions $\mathrm{P} 1 \mathrm{a}, \mathrm{P} 2 \mathrm{~b}$, and P3 were each mutated individually. The wild-type and mutated sequences (in bold letters) are depicted with upper (up) and lower (dn) strands oriented as shown in Figure 1A. In mutant P3-up $\Delta$, all 10 bases of the upper P3 strand were deleted. $(B)$ Complementary P3 mutants dimerize. Shown is a representative experiment, performed as in $A$, with P3 mutant RNAs present singly or mixed as indicated. $(C)$ The extent of dimerization (dimers as a percentage of total input radiolabeled RNA, quantified by phosphorimaging) was determined as described above for the P3-A-up and P3-A-dn mutants individually, in cis, and at equimolar concentration in trans. The data shown are mean \pm standard error for six independent determinations. 
Ly et al.

mutations were combined in the same RNA molecule (i.e., in cis) so as to restore P3 complementarity, dimerization was restored to essentially wild-type levels in vitro (Fig. 2B). Notably, dimerization was also fully restored when the same two mutations were combined in trans - that is, on two separate RNA molecules in an equimolar mixture (Fig. 2B). Thus, two complementary P3-mutant RNAs that cannot form dimers or pseudoknots individually dimerized readily when combined. These results indicate that intermolecular complementarity of the P3 region can lead to efficient hTER dimerization in vitro, as predicted if P3 base-pairing in trans creates at least part of the dimer interface.

We then tested the effects of P3 trans-complementarity on telomerase enzymatic activity in two different systems. To reconstitute telomerase in vitro, hTERT protein was produced in a rabbit reticulocyte lysate (RRL) to which synthetic wild-type or mutant hTERs were also added (Beattie et al. 2001). The ability of the resulting complexes to add telomeric DNA repeats onto a synthetic DNA primer was then measured in a semiquantitative telomerase activity (TRAP) assay (Fig. 3A). The P3-A-up and P3-A-dn mutations, when present individually, each showed only trace catalytic function $(<0.1 \%$ of wild type). Activity was reproducibly enhanced when both mutations were combined in cis and, strikingly, nearly the same degree of enhancement was also observed when we combined the two mutations in trans, using half the amount of each RNA so that total input RNA remained constant for any given RNA dilution. The results were the same regardless of whether the P3-up and P3-dn mutant hTER RNAs were mixed together prior to their addition to the hTERT expressed in the RRL system, or whether each hTER mutant was first added to the RRL-expressed hTERT, incubated for $1 \mathrm{~h}$, and then the two reconstituted telomerase preparations were mixed and incubated for another hour before assaying for telomerase as above.

To determine whether the P3 mutant telomerase RNAs retained the ability to coimmunoprecipitate with hTERT protein, the wild-type and mutant RNAs were each incubated with Flag-epitope-tagged hTERT, then immunoprecipitated by a Flag-specific antibody, and the precipitated complexes were probed for hTER by Northern blotting. None of the P3 pairing mutants interfered with hTERT-binding (Supplementary Fig. 2A). Hence, defects in P3 pairing influence enzymatic activity without eliminating the interaction of hTER with hTERT.

These results were confirmed and extended by reconstituting telomerase activity in vivo using human VA13 fibroblasts. These cells have no detectable endogenous hTERT protein or hTER (Bryan et al. 1995), but when transfected with wild-type hTERT and hTER expression constructs can assemble functional telomerase complexes. We transiently transfected VA13 cells with a vector encoding wild-type hTER or various P3 mutants along with a second vector construct that expressed hTERT protein, then measured telomerase activity in cell lysates prepared $48 \mathrm{~h}$ after transfection. Northern blots of these lysates confirmed that all the P3 mutant hTERs were stably expressed at levels comparable to that of wild-type hTER (Supplementary Fig. 2B). TRAP assays of the lysates, however, revealed virtually no detectable telomerase activity in cells transfected with either P3-A-up or P3-A-dn alone (Fig. 3B, "up" and "dn"

A

A wild

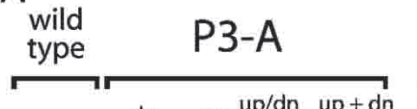

B
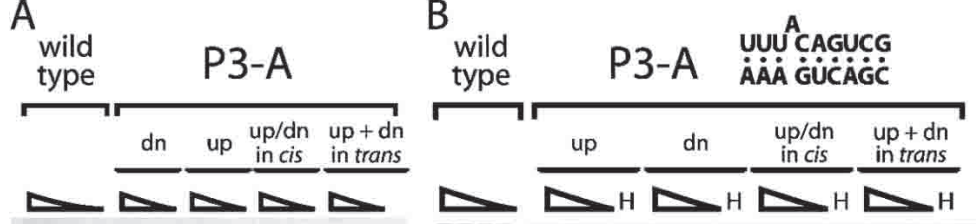

C
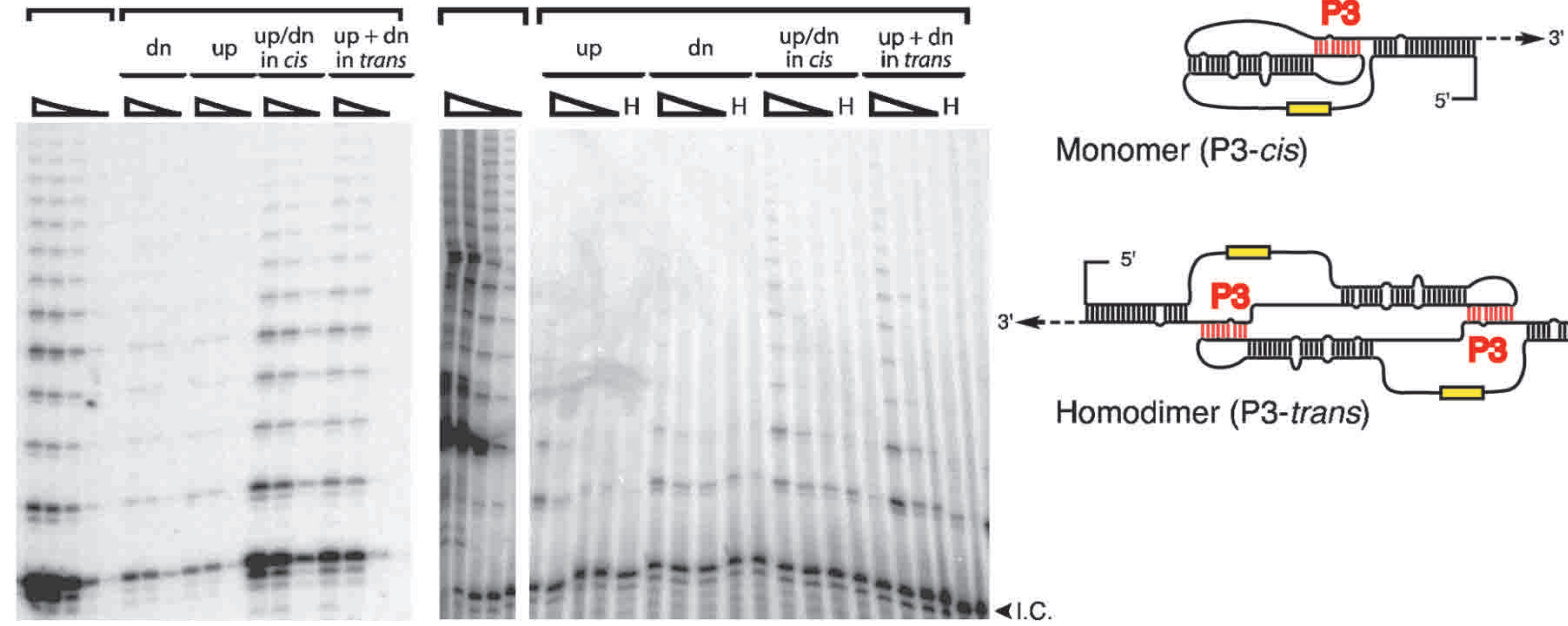

Monomer (P3-cis)

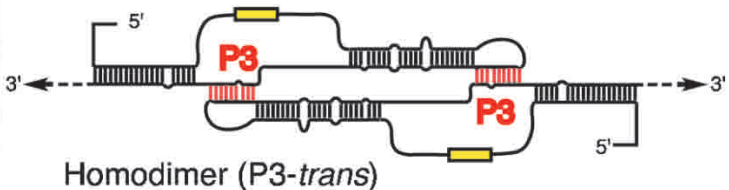

Figure 3. Dimerization restores biological activity of inactive hTER P3 mutant pairs assembled with hTERT. $(A)$ Telomerase activity was reconstituted in vitro with varying amounts of wild-type or P3-A mutant forms of hTER (see Materials and Methods). The telomere repeat amplification protocol (TRAP) assays were performed with the lysates. Triangles denote sequential 10-fold dilutions of input lysate. Products were analyzed by denaturing gel electrophoresis and phosphorimaging. The data shown are representative of three replicate assays using two independent preparations of expression vector encoding each mutant RNA. (B) In vivo activity of wild-type and P3 mutants. The Flag-epitopetagged hTERT and hTER constructs were transfected into VA13 cells. TRAP assays were performed on cell extracts. The triangles at top indicate sequential fivefold dilutions of the input whole-cell extract; " $\mathrm{H}^{\prime \prime}$ indicates that undiluted cell extract was heat-inactivated at $85^{\circ} \mathrm{C}$ for 5 min prior to TRAP assay. All wild-type and P3-A lanes are from the same gel and autoradiogram. The data shown are representative of three or more replicate assays using at least two independent preparations of expression vector encoding each mutant RNA. "I.C." indicates PCR products of internal control included for normalization of PCR efficiency. (C) Cis and trans conformations of the hTER pseudoknot domain. The 3' half of each hTER RNA is omitted (broken arrow). Yellow indicates templating domain; red indicates P3 structures. 
lanes); the finding that these mutants appeared more severely defective in cells than in the in vitro reconstitution system (Fig. 3A) may reflect the far lower concentrations of hTER achievable in the cell-based assay. Nevertheless, as shown in Figure 3B, combining the two P3-A mutants in trans (as well as in cis) again yielded a reproducibly higher level of telomere repeat synthesis than was produced by either mutant individually. To ensure that the phenomenon was not unique to those mutants, we also designed a second pair (called P3-B-up and P3-B-dn) that each differed from their P3-A counterparts at two base positions (Supplementary Fig. 3). These complementary P3-B mutants, too, were barely active when tested singly, but showed a small but reproducible increase in activity when coexpressed in trans (Supplementary Fig. 3).

Possible intermolecular RNA-RNA interactions within the active telomerase complex have not been previously explored. Our study reveals that hTER can undergo P3-dependent homodimerization to achieve a novel RNA "trans-pseudoknot" conformation that we term P3-trans (Fig. 3C). We demonstrated that wild-type hTER self-assembles efficiently into dimers in vitro through a reaction that requires interstrand complementarity at the P3 locus. This loop-mediated homodimerization closely mimics that observed in all retroviral genomes studied to date (Laughrea and Jette 1994; Paillart et al. 1996; Clever and Parslow 1997; Ly and Parslow 2002), as well as in two cellular RNAs (Wagner et al. 2001; Wittenhagen and Kelley 2002). By creating obligate dimers of inactive hTER mutant pairs whose P3 sequences could support trans but not cis base-pairing, we also have shown that the hTER dimer supports telomerase function both in rabbit reticulocyte lysates and in human cells. Our results further indicate that defects in P3-mediated dimerization are associated with a loss of hTER enzymatic activity rather than an inability to assemble with hTERT (Supplementary Fig. 2A), a finding compatible with other reports that pseudoknot mutations do not appreciably affect holoenzyme assembly when telomerase RNA is in excess (Mitchell and Collins 2000; Bachand and Autexier 2001; Beattie et al. 2001; Martin-Rivera and Blasco 2001).

The mammalian telomerase holoenzyme is a multimeric complex of $1000 \mathrm{kD}$ or more whose composition is not fully known (Nakamura et al. 1997; Schnapp et al. 1998; Bachand et al. 2001; Blasco 2002). After salt-stripping to remove weakly interacting proteins, however, the smallest human telomerase complex that retains enzymatic activity has an apparent mass near $600 \mathrm{kD}$, consistent with its containing two copies each of hTER and hTERT (Wenz et al. 2001). Physical oligomerization of hTERT has been reported to be necessary, though not sufficient, for enzymatic activity (Moriarty et al. 2002). P3-mediated dimerization of the type we propose here could provide either the scaffold for assembling the holoenzyme complex or RNA-RNA interactions necessary for its activity.

Our results provide the first information about a novel mode of RNA-RNA interaction, the P3-trans pairing, that we have shown here can be made obligatory for enzymatic activity. Our findings do not address the relative contributions of monomeric and dimeric hTER to normal wild-type telomerase function. Notably, however, both pairs of mutants tested gave comparable levels of activity in trans as in cis. The individual P3-up or
P3-dn mutants used in this study have multiple substitutions in P3 that, when present by themselves, are incompatible with either intramolecular or trans-pseudoknot formation. When tested individually, each caused severe functional and dimerization defects. Even when combined to restore P3 base-pairing, these extensively mutated RNAs regained only a small fraction of wildtype activity level, consistent with the fact that the P3 primary sequence is very strongly conserved among vertebrates (Chen et al. 2000). As indicated by chemical accessibility mapping and NMR structural data (Bhattacharyya and Blackburn 1994; Zaug and Cech 1995; Antal et al. 2002; Comolli et al. 2002; Theimer et al. 2003), the P3 pairing may be dynamic, and need to attain a balance between different conformations, perhaps adopted through each cycle of telomere repeat synthesis or in various physiological states.

Direct P3-mediated dimer interactions may account for the previous finding that particular telomerase RNA mutants can functionally inhibit (Martin-Rivera and Blasco 2001; Wenz et al. 2001), and that others are activated by (Prescott and Blackburn 1997a), coexpressed wild-type RNA. They are also consistent with the finding (Tesmer et al. 1999) that two different fragments of wild-type hTER that each contain only one strand of P3 can combine to form a functional telomerase, as these fragments would each be expected to bind hTERT independently (Mitchell and Collins 2000) and together could simulate the P3-trans linkage. Although hTERT protein can multimerize in vitro without RNA (Arai et al. 2002), pairs of inactive hTERT mutants are unable to complement each other functionally unless hTER is present and both mutants retain hTER-binding activity (Moriarty et al. 2002). Changes in dimer RNA conformation, transmitted across the P3-trans interface, could enable one hTERT molecule to influence another allosterically, as has been proposed (Beattie et al. 2001; Moriarty et al. 2002), and physically linking two templates in this manner could also give rise to the dense admixture of telomere repeat sequences produced by both yeast and ciliate cells that coexpress mutant and wild-type telomerase RNAs (Yu and Blackburn 1991; Prescott and Blackburn 1997b).

TERT and retroviral RTs likely share a common ancestor (Nakamura and Cech 1998; Peng et al. 2001). Our results suggest that the parallels extend further in that each class of enzyme uses a homodimeric RNA as its natural template. For retroviruses, whose RTs have a single active site and must copy long genomic RNAs in their entirety, frequent switching between duplicate templates makes it possible to circumvent breaks in the individual RNA strands that would otherwise be lethal, and also provides the principal mechanism of genetic recombination (Coffin 1979; Hu and Temin 1990). Telomerases, in contrast, have evolved to use a unique, short, internal template sequence for repeated cycles of DNA synthesis. In that context, hTER dimerization may provide a means to sustain the association between a telomerase holoenzyme and its product between cycles: if one template region in the dimer remains annealed to the newly synthesized repeat, as has been shown in vitro for two yeast telomerases (Prescott and Blackburn 1997b; Fulton and Blackburn 1998; Wenz et al. 2001), it could make the newly elongated end readily available to its partner active site for the next cycle, enabling the RNA dimer with its associated TERT proteins to translocate 
in a processive, ratcheting motion at the growing chromosomal terminus (Supplementary Fig. 4). Alternatively, like replicative DNA polymerases and other replicase complexes that have dual active sites, telomerases may have evolved to act on two nucleic acid strands simultaneously in a coordinated fashion. For telomerase, these might include pairs of sister- or non-sister-chromatid telomeres newly replicated in mitosis or meiosis, or chromosomal ends brought together near the periphery of interphase nuclei (Supplementary Fig. 4). In those settings, an hTER dimer at the core of telomerase could be viewed as an RNA bridge linking the $5^{\prime}$ ends of two chromosomes.

\section{Materials and methods}

In vitro hTER RNA dimerization assay

Synthetic RNAs representing the indicated portions of hTER were denatured in water at $95^{\circ} \mathrm{C}$ for $3 \mathrm{~min}$, immediately snap-cooled on ice, and adjusted to $50 \mathrm{mM} \mathrm{NaCl}, 25 \mathrm{mM}$ Tris (pH 7.0), $10 \mathrm{mM} \mathrm{MgCl}_{2}$, and then either kept on ice $(-)$ or prewarmed at $37^{\circ} \mathrm{C}(+)$ for $2 \mathrm{~h}$ before analysis by nondenaturing gel electrophoresis in $90 \mathrm{mM}$ Tris-borate, $0.1 \mathrm{mM} \mathrm{MgCl}_{2}$. Each $10-\mu \mathrm{L}$ dimerization reaction contained $10^{4} \mathrm{cpm}$ of tracer ${ }^{32} \mathrm{P}$-labeled hTER and $1 \mu \mathrm{g}$ of the same RNA in unlabeled form.

In vitro and in vivo reconstitutions of telomerase enzymatic assays Reconstitution of telomerase activity was performed as described (Beattie et al. 2000; see Supplemental Material).

\section{Acknowledgments}

We thank J. Clever, Y. Liang, and S. Nautiyal for comments on the manuscript. Supported by NIH grants AI40317 and AI36636 to T.G.P. and GM26259 to E.H.B. H.L. was supported by UCSF AIDS postdoctoral training grant AI07395 and a special fellowship from the Leukemia and Lymphoma Society of America, L.X. was supported by a Susan G. Komen postdoctoral fellowship, and M.A.R. was supported by an NSF predoctoral fellowship.

The publication costs of this article were defrayed in part by payment of page charges. This article must therefore be hereby marked "advertisement" in accordance with 18 USC section 1734 solely to indicate this fact.

\section{References}

Antal, M., Boros, E., Solymosy, F., and Kiss, T. 2002. Analysis of the structure of human telomerase RNA in vivo. Nucleic Acids Res. 30: 912-920.

Arai, K., Masutomi, K., Khurts, S., Kaneko, S., Kobayashi, K., and Murakami, S. 2002. Two independent regions of human telomerase reverse transcriptase are important for its oligomerization and telomerase activity. J. Biol. Chem. 277: 8538-8544.

Bachand, F. and Autexier, C. 2001. Functional regions of human telomerase reverse transcriptase and human telomerase RNA required for telomerase activity and RNA-protein interactions. Mol. Cell. Biol. 21: 1888-1897.

Bachand, F., Triki, I., and Autexier, C. 2001. Human telomerase RNAprotein interactions. Nucleic Acids Res. 29: 3385-3393.

Beattie, T.L., Zhou, W., Robinson, M.O., and Harrington, L. 2000. Polymerization defects within human telomerase are distinct from telomerase RNA and TEP1 binding. Mol. Biol. Cell 11: 3329-3340.

- 2001. Functional multimerization of the human telomerase reverse transcriptase. Mol. Cell. Biol. 21: 6151-6160.

Bhattacharyya, A. and Blackburn, E.H. 1994. Architecture of telomerase RNA. EMBO J. 13: 5721-5723.

Blackburn, E.H. 2000. The end of the (DNA) line. Nat. Struct. Biol. 7: 847-850.

Blasco, M.A. 2002. Immunosenescence phenotypes in the telomerase knockout mouse. Springer Semin. Immunopathol. 24: 75-85.

Bryan, T.M., Englezou, A., Gupta, J., Bacchetti, S., and Reddel, R.R. 1995.
Telomere elongation in immortal human cells without detectable telomerase activity. EMBO J. 14: 4240-4248.

Chen, J.L., Blasco, M.A., and Greider, C.W. 2000. Secondary structure of vertebrate telomerase RNA. Cell 100: 503-514.

Chen, J.L., Opperman, K.K., and Greider, C.W. 2002. A critical stem-loop structure in the CR4-CR5 domain of mammalian telomerase RNA. Nucleic Acids Res. 100: 503-514.

Clever, J.L. and Parslow, T.G. 1997. Mutant human immunodeficiency virus type 1 genomes with defects in RNA dimerization or encapsidation. J. Virol. 71: 3407-3414.

Coffin, J.M. 1979. Structure, replication, and recombination of retrovirus genomes: Some unifying hypotheses. J. Gen. Virol. 42: 1-26.

Comolli, L.R., Smirnov, I., Xu, L., Blackburn, E.H., and James, T.L. 2002. A molecular switch underlies a human telomerase disease. Proc. Nat1. Acad. Sci. 99: 16998-17003.

Fulton, T.B. and Blackburn, E.H. 1998. Identification of Kluyveromyces lactis telomerase: Discontinuous synthesis along the 30-nucleotidelong templating domain. Mol. Cell. Biol. 18: 4961-4970.

Gilley, D. and Blackburn, E.H. 1999. The telomerase RNA pseudoknot is critical for the stable assembly of a catalytically active ribonucleoprotein. Proc. Natl. Acad. Sci. 96: 6621-6625.

Hu, W.S. and Temin, H.M. 1990. Genetic consequences of packaging two RNA genomes in one retroviral particle: Pseudodiploidy and high rate of genetic recombination. Proc. Natl. Acad. Sci. 87: 1556-1560.

Laughrea, M. and Jette, L. 1994. A 19-nucleotide sequence upstream of the $5^{\prime}$ major splice donor is part of the dimerization domain of human immunodeficiency virus 1 genomic RNA. Biochemistry 33: 1346413474

Lingner, J., Hughes, T.R., Shevchenko, A., Mann, M., Lundblad, V., and Cech, T.R. 1997. Reverse transcriptase motifs in the catalytic subunit of telomerase. Science 276: 561-567.

Ly, H. and Parslow, T.G. 2002. Bipartite signal for genomic RNA dimerization in Moloney murine leukemia virus. J. Virol. 76: 3135-3144.

Martin-Rivera, L. and Blasco, M.A. 2001. Identification of functional domains and dominant negative mutations in vertebrate telomerase RNA using an in vivo reconstitution system. I. Biol. Chem. 276: 5856-5865.

Mitchell, J.R. and Collins, K. 2000. Human telomerase activation requires two independent interactions between telomerase RNA and telomerase reverse transcriptase. Mol. Cell 6: 361-371.

Moriarty, T.J., Huard, S., Dupuis, S., and Autexier, C. 2002. Functional multimerization of human telomerase requires an RNA interaction domain in the $\mathrm{N}$ terminus of the catalytic subunit. Mol. Cell. Biol. 22: $1253-1265$.

Nakamura, T.M. and Cech, T.R. 1998. Reversing time: Origin of telomerase. Cell 92: 587-590.

Nakamura, T.M., Morin, G.B., Chapman, K.B., Weinrich, S.L., Andrews, W.H., Lingner, J., Harley, C.B., and Cech, T.R. 1997. Telomerase catalytic subunit homologs from fission yeast and human. Science 277: 955-959.

Nugent, C.I. and Lundblad, V. 1998. The telomerase reverse transcriptase: Components and regulation. Genes \& Dev. 12: 1073-1085.

Paillart, J.C., Skripkin, E., Ehresmann, B., Ehresmann, C., and Marquet, R. 1996. A loop-loop 'kissing' complex is the essential part of the dimer linkage of genomic HIV-1 RNA. Proc. Nat1. Acad. Sci. 93: 5572-5577.

Peng, Y., Mian, I.S., and Lue, N.F. 2001. Analysis of telomerase processivity: Mechanistic similarity to HIV-1 reverse transcriptase and role in telomere maintenance. Mol. Cell 7: 1201-1211.

Prescott, J. and Blackburn, E.H. 1997a. Functionally interacting telomerase RNAs in the yeast telomerase complex. Genes \& Dev. 11: 2790-2800.

- 1997b. Telomerase RNA mutations in Saccharomyces cerevisiae alter telomerase action and reveal nonprocessivity in vivo and in vitro. Genes \& Dev. 11: 528-540.

Schnapp, G., Rodi, H.P., Rettig, W.J., Schnapp, A., and Damm, K. 1998. One-step affinity purification protocol for human telomerase. Nucleic Acids Res. 26: 3311-3313.

Tesmer, V.M., Ford, L.P., Holt, S.E., Frank, B.C., Yi, X., Aisner, D.L. Ouellette, M., Shay, J.W., and Wright, W.E. 1999. Two inactive fragments of the integral RNA cooperate to assemble active telomerase with the human protein catalytic subunit (hTERT) in vitro. Mol. Cell. Biol. 19: 6207-6216. 
Theimer, C.A., Finger, L.D., Trantirek L., and Feigon J. 2003. Mutations linked to dyskeratosis congenita cause changes in the structural equilibrium in telomerase RNA. Proc. Nat1. Acad. Sci. 100: 449-454.

Vulliamy, T., Marrone, A., Goldman, F., Dearlove, A., Bessler, M., Mason, P.J., and Dokal, I. 2001. The RNA component of telomerase is mutated in autosomal dominant dyskeratosis congenita. Nature 413: 432-435.

Wagner, C., Palacios, I., Jaeger, L., St Johnston, D., Ehresmann, B., Ehresmann, C., and Brunel, C. 2001. Dimerization of the 3'UTR of bicoid mRNA involves a two-step mechanism. J. Mol. Biol. 313: 511-524.

Wenz, C., Enenkel, B., Amacker, M., Kelleher, C., Damm, K., and Lingner, J. 2001. Human telomerase contains two cooperating telomerase RNA molecules. EMBO J. 20: 3526-3534.

Wittenhagen, L.M. and Kelley, S.O. 2002. Dimerization of a pathogenic human mitochondrial tRNA. Nat. Struct. Biol. 9: 586-590.

Yu, G.L. and Blackburn, E.H. 1991. Developmentally programmed healing of chromosomes by telomerase in Tetrahymena. Cell 67: 823832.

Zaug, A.J. and Cech, T.R. 1995. Analysis of the structure of Tetrahymena nuclear RNAs in vivo: Telomerase RNA, the self-splicing rRNA intron, and U2 snRNA. RNA 1: 363-374. 


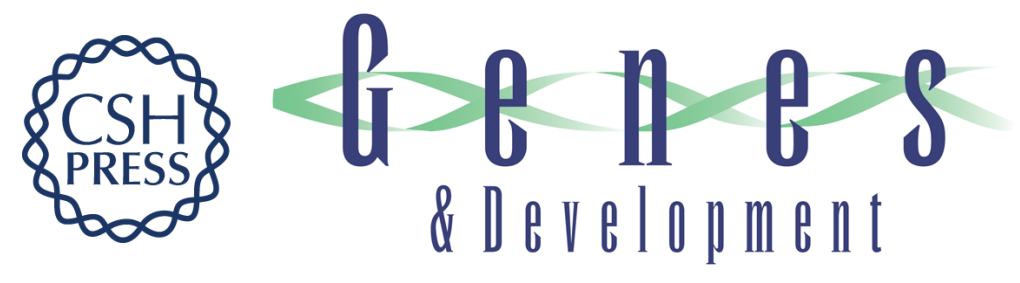

\section{A role for a novel 'trans-pseudoknot' RNA-RNA interaction in the functional dimerization of human telomerase}

Hinh Ly, Lifeng Xu, Melissa A. Rivera, et al.

Genes Dev. 2003, 17:

Access the most recent version at doi:10.1101/gad.1060803

Supplemental http://genesdev.cshlp.org/content/suppl/2003/05/04/17.9.1078.DC1
Material

References This article cites 40 articles, 22 of which can be accessed free at:

http://genesdev.cshlp.org/content/17/9/1078.full.html\#ref-list-1

License

Email Alerting Receive free email alerts when new articles cite this article - sign up in the box at the top

Service

right corner of the article or click here.

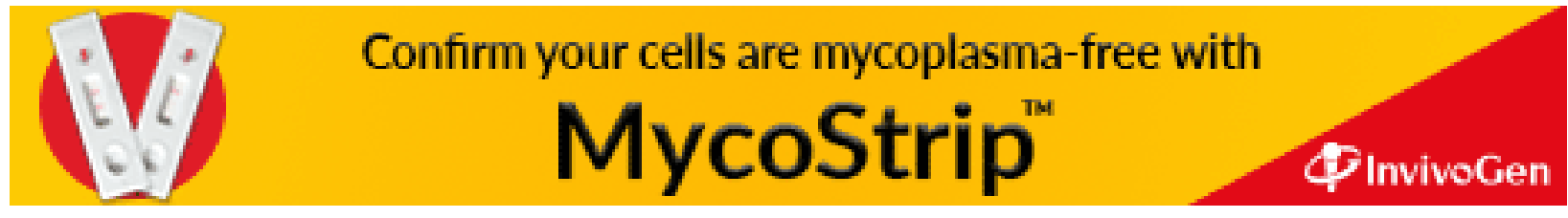

